\title{
The effects of intraoperative dexamethasone on left atrial function and postoperative atrial fibrillation in cardiac surgical patients
}

\author{
K. A. Jacob • J. M. Dieleman • H. M. Nathoe • D. van Osch • \\ E. E. C. de Waal • M. J. Cramer • J. Kluin • D. van Dijk
}

(C) The Author(s) 2014. This article is published with open access at Springerlink.com

\begin{abstract}
Postoperative new-onset atrial fibrillation (PNAF) is very common after cardiac surgery and postoperative inflammation may contribute to PNAF by inducing atrial dysfunction. Corticosteroids reduce inflammation and may thus reduce atrial dysfunction and PNAF development. This study aimed to determine whether dexamethasone protects against left atrial dysfunction and PNAF in cardiac surgical patients. Cardiac surgical patients were randomised to a single dose of dexamethasone $\left(1 \mathrm{mg} \cdot \mathrm{kg}^{-1}\right)$ or placebo after inducing anaesthesia. Transoesophageal echocardiography was performed in patients before and after surgery. Primary outcome was left atrial total ejection fraction (LA-TEF) after sternal closure; secondary outcomes included left atrial diameter and PNAF. 62 patients were included. Baseline characteristics were well balanced. Postoperative LA-TEF was $36.4 \%$ in the dexamethasone group and $40.2 \%$ in the placebo group (difference $-3.8 \%$; $95 \%$ confidence interval $(\mathrm{CI})-9.0$ to $1.4 \% ; P=$ 0.15). Postoperative left atrial diameter was 4.6 and $4.3 \mathrm{~cm}$, respectively (difference $0.3 ; 95 \% \mathrm{CI}-0.2$ to $0.7 ; P=0.19$ ). The incidence of PNAF was $30 \%$ in the dexamethasone group and $39 \%$ in the placebo group $(P=0.47)$. Intraoperative high-dose dexamethasone did not protect against postoperative left atrial dysfunction and did not reduce the risk of PNAF in cardiac surgical patients.
\end{abstract}

K. A. Jacob $(\bowtie) \cdot$ J. M. Dieleman • E. E. C. de Waal • D. van Dijk Department of Anesthesiology and Intensive Care, University Medical Center Utrecht, Mail Stop F.06.149, PO Box 85500, 3508 Utrecht, the Netherlands

e-mail: k.a.jacob@umcutrecht.nl

K. A. Jacob $\cdot$ J. Kluin

Department of Cardiothoracic Surgery, University Medical Center

Utrecht, Utrecht, the Netherlands

H. M. Nathoe · D. van Osch • M. J. Cramer

Department of Cardiology, University Medical Center Utrecht,

Utrecht, the Netherlands
Keywords Atrial fibrillation · Cardiac surgery · Left atrial ejection fraction $\cdot$ Left atrial dimension $\cdot$ Corticosteroids

\section{Introduction}

Postoperative new-onset atrial fibrillation (PNAF) is a complication occurring in $25-30 \%$ of cardiac surgical patients. PNAF may lead to significant morbidity, including stroke, renal and respiratory failure, and is associated with an increased mortality [1-3].

Several preoperative echocardiographic findings have been implicated as predictors of PNAF. These predictive measures include increased left atrial (LA) volume and dysfunction [4-9]. A recent study showed that decreased LA total ejection fraction (TEF) was the most potent predictor of PNAF [10].

The underlying pathophysiological mechanisms are complex. Apart from pre-existing atrial dysfunction, the perioperative systemic inflammatory response may play a role in affecting atrial function, along with atrial myocardial injury and ischaemia [11-13]. The importance of inflammatory mechanisms is supported by several studies, demonstrating that atrial biopsies taken during cardiac surgery show marked oxidative damage and interstitial fibrosis [11, 13, 14].

The effects of administration of corticosteroids on the perioperative inflammatory response during cardiac surgery have been extensively investigated. Corticosteroids are thought to decrease the levels of serum inflammatory markers [15], reduce the risk of postoperative respiratory failure, and shorten the duration of postoperative intensive care unit and hospital stay [16, 17]. Furthermore, steroids may reduce the incidence of PNAF. Although the latter effect seems related to the attenuated inflammatory response [18], the exact mechanisms are unclear.

This study aimed to evaluate the effect of dexamethasone administration on postoperative LA dysfunction and 
dilatation. We hypothesised that high-dose dexamethasone increases LA-TEF, decreases postoperative LA diameter and protects against PNAF.

\section{Materials and methods}

Study design and patient selection

The DECS-PNAF study was a substudy of the Dexamethasone for Cardiac Surgery (DECS) study. It was a pre-planned and prospectively designed and performed substudy in which patients with a minimum age of 18 years, undergoing cardiac surgery, were enrolled in this multicentre randomised, double-blind, placebo-controlled study, comparing high-dose intravenous dexamethasone with placebo treatment (trial registration: ClinicalTrials.gov, number NCT00293592) [16]. DECS study patients in one of the participating centres, i.e. University Medical Centre Utrecht, who were scheduled for coronary artery bypass grafting $(\mathrm{CABG})$, aortic valve surgery, or a combination of these procedures, without other concomitant valve surgery or other additional procedures, were eligible for inclusion in this DECS-PNAF study. Patients with either a history of atrial fibrillation, a contraindication for intraoperative transoesophageal echocardiography, or a preoperative cardiac rhythm other than sinus rhythm, were not eligible for inclusion.

The research ethics committee of the University Medical Center Utrecht approved the research protocol. All patients provided written informed consent before randomisation. The study was performed in accordance with the Medical Research Involving Human Subjects Act (WMO) and institutional regulations and guidelines.

\section{Intervention}

Patients were randomised to receive either dexamethasone or placebo treatment. Dexamethasone $\left(1 \mathrm{mg} \cdot \mathrm{kg}^{-1}\right.$ with a maximum of $100 \mathrm{mg}$ ) or placebo was administered as a single intravenous injection after induction of anaesthesia, but before commencement of cardiopulmonary bypass. Comprehensive information about the randomisation methods has been published previously [16].

\section{Primary and secondary outcomes}

The primary endpoint of this study was the LA-TEF at the end of the surgical procedure. Secondary endpoints included LA diameter, LA area, maximum and minimum LA volume in the cardiac cycle, left atrial appendage (LAA) area and velocity and left pulmonary vein systolic/diastolic (S/D) ratio at the same time point. We also evaluated the occurrence of PNAF during the first 5 days after surgery.

Data collection

Baseline patient characteristics and surgical characteristics were prospectively collected as part of the DECS study [16]. For the present substudy, patients underwent transoesophageal echocardiography after induction of anaesthesia, and after sternal closure at the end of the operation, while the patient was in a supine position. The required images were stored and analysed offline by two independent blinded observers, using the Philips XCELERA Cardiology image (Version 3.2) software package. All measurements were done twice and averaged.

LA diameter was measured from the posterior LA wall to the mitral annulus, at the end of ventricular systole in the midoesophageal long-axis view with the transducer array at $\sim 120^{\circ}$ $[19,20]$. LA volume was assessed by the area-length method from both the mid-oesophageal four-chamber and twochamber views, with LA size being optimised in both views. Biplane LA volume was calculated using Simpson's method. The LA volume was measured at both the end of diastole (left atrial end-diastolic volume, being maximum LAV), and at the end of systole (left atrial end-systolic volume, being minimum LAV) [20]. LA-TEF was calculated using the following formula: (((LAVmax - LAVmin)/LAVmax $) \times 100 \%)$. In patients in whom the entire dimensions or volume of the LA were not visible in the scan sector, measurements were traced to the edge of the field of view. The LAA was imaged in the midoesophageal two-chamber view. The LAA area was calculated using Simpson's method, at the end of ventricular systole, and peak velocity was measured by pulse wave Doppler, with the pulsed Doppler sample volume placed in the LAA cavity. Peak flow velocity in the left pulmonary vein was recorded and measured using a biphasic systolic and diastolic velocity wave. The S/D ratio was calculated afterwards.

The occurrence of PNAF was evaluated with continuous Holter monitoring (leads V1-V5) for the first 5 days postoperatively in all patients. The occurrence of PNAF was defined as the occurrence of any episode of atrial fibrillation or atrial flutter during any of these five postoperative days.

Statistical analysis

Normally distributed continuous variables are presented as means with standard deviations $( \pm \mathrm{SD})$. Data in both groups were compared using an independent $t$-test. Continuous data that were not normally distributed are presented as medians with interquartile range and were compared using a MannWhitney $U$-test. Dichotomous characteristic variables are presented as absolute numbers with percentages and were compared using a $\chi^{2}$ test. We calculated mean differences with 
$95 \%$ confidence intervals (CIs), for continuous and dichotomous outcomes, respectively. In addition to the analyses based on the randomisation to dexamethasone or placebo, we evaluated the association between LATEF and PNAF using an independent $t$-test. A $p$-value $<$ 0.05 was considered significant in the analysis models. Statistical analysis was performed using IBM SPSS version 20.0 (SPSS Inc., Chicago, IL, USA).

\section{Results}

The DECS-PNAF transoesophageal echocardiography substudy included 62 patients (Fig. 1). Patients in the two study groups were comparable with no statistical differences in baseline demographic, clinical, and surgical aspects (Table 1).

At the beginning of the operation, after induction of anaesthesia, echocardiographic data were collected in only 22 patients in each group. This is because preoperative ultrasound was not routinely assessed in all patients, as was the case with postoperative ultrasound. There were no differences in any of the obtained echocardiographic parameters between the dexamethasone group and the placebo group (Table 2). Also, no differences were found in preoperative and postoperative LA dimensions and function between patients undergoing valve surgery or CABG in both the placebo and dexamethasone group.
Primary outcome

The mean LA-TEF after sternal closure was $36.4 \%( \pm 11.9 \%)$ in the dexamethasone group and $40.2 \%( \pm 7.9 \%)$ in the placebo group (mean difference $-3.8 \%$; $95 \% \mathrm{CI}-9.0$ to $1.4 \% ; P=0.15$ ) (Table 3$)$.

Secondary outcomes

After sternal closure, there were no differences in LA diameter, LA area, maximum and minimum volume of the left atrium, LAA area and velocity, and left pulmonary S/D ratio between the two groups (Table 3 ).

PNAF occurred in 21 of the 62 patients (34\%) and was most commonly detected on the third postoperative day, with a median duration ofl day. In the dexamethasone group, nine patients developed PNAF (30\%), while 12 patients developed PNAF in the placebo group $(39 \%)(P=0.47)$. In patients who developed PNAF, mean LA-TEF was $38.6 \%( \pm 9.1 \%)$, as compared with $38.0 \%( \pm 10.9 \%)$ in patients in whom PNAF did not occur (mean difference $0.6 ; 95 \% \mathrm{CI}$ : -4.6 to $5.9 ; P=$ $0.81)$.

\section{Discussion}

In this study we evaluated the effect of high-dose intraoperative dexamethasone on echocardiographic measures of LA
Fig. 1 Enrolment flowchart. In the 62 patients included for postoperative analysis. TOE: transoesophageal

echocardiography

\section{Enrollment Flowchart}

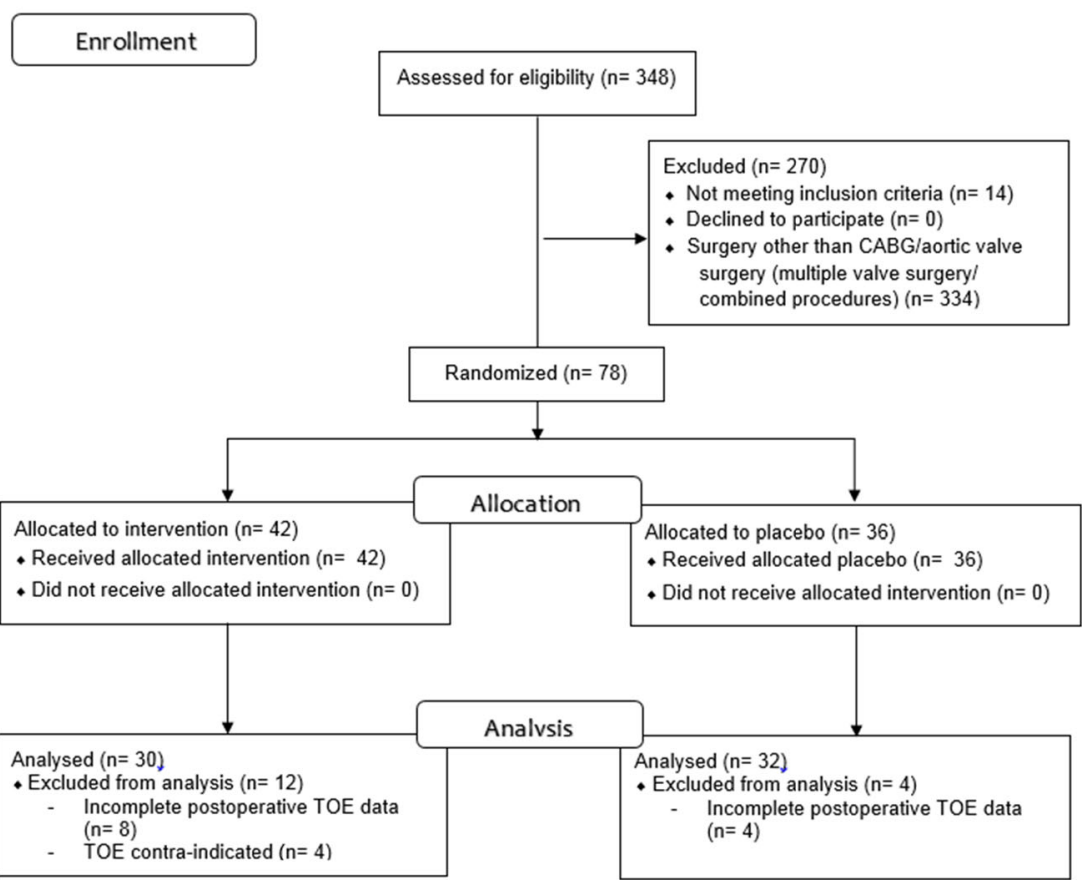


Table 1 Demographic, clinical, and surgical characteristics of the dexamethasone and placebo groups $^{\alpha}$
Abbreviations: ACEI: ACEinhibitors; $A R B s$ : angiotensin II receptor blockers; $B S A$ : body surface area; $C A B G$ : coronary artery bypass graft; $C P B$ : cardiopulmonary bypass; $I Q R$, interquartile range; $N$ : number. $S I$ conversion: To convert creatinine to $\mathrm{mg} / \mathrm{dL}$, multiply by 0.0113

${ }^{\alpha}$ Data are shown as N (\%) unless otherwise specified

${ }^{\beta}$ BSA calculated using the Haycock formula

$\gamma$ Definition of left ventricular function class: good, ejection fraction of $>50 \%$, moderate, ejection fraction of 30-50\%; and poor, ejection fraction of $<30 \%$

${ }^{\delta}$ Higher EuroScores present increased risk of perioperative mortality

¿ Valve surgery includes aortic valve surgery

\begin{tabular}{|c|c|c|}
\hline Characteristics & Dexamethasone $N=30$ & Placebo $N=32$ \\
\hline \multicolumn{3}{|l|}{ Demographics } \\
\hline Age, mean (SD), years & $70.4(9.1)$ & $68.9(9.0)$ \\
\hline Male sex & $24(80)$ & $23(72)$ \\
\hline BMI, mean (SD), $\mathrm{kg} / \mathrm{m}^{2}$ & $27.96(4.79)$ & $26.83(3.28)$ \\
\hline BSA, mean (SD), $\mathrm{m}^{2},{ }^{\beta}$ & $2.04(0.20)$ & $1.97(0.17)$ \\
\hline \multicolumn{3}{|l|}{ Coexisting medical conditions } \\
\hline Hypertension & $18(60)$ & $20(63)$ \\
\hline Diabetes mellitus & $7(23)$ & $3(9)$ \\
\hline Treatment for pulmonary disease & $2(7)$ & $7(22)$ \\
\hline Peripheral vascular disease & $5(17)$ & $8(25)$ \\
\hline Preoperative creatinine, mean (SD), $\mu \mathrm{mol} / 1$ & $89.3(18.6)$ & $92.8(18.6)$ \\
\hline Recent myocardial infarction & $6(20)$ & $5(16)$ \\
\hline Left ventricular function, Good, $\gamma$ & $21(70)$ & $21(66)$ \\
\hline EuroScore, median (IQR)c, ${ }^{\delta}$ & $5(4-7)$ & $5(3-7)$ \\
\hline \multicolumn{3}{|l|}{ Preoperative medication } \\
\hline Statin & $28(93)$ & $22(69)$ \\
\hline Beta blocker & $22(73)$ & $20(63)$ \\
\hline Diuretics & $8(27)$ & $8(25)$ \\
\hline Aspirin & $23(77)$ & $24(75)$ \\
\hline Calcium entry blockers & $25(83)$ & $21(66)$ \\
\hline Nitrates & $15(50)$ & $11(34)$ \\
\hline ACEI/ARBs & $18(60)$ & $13(41)$ \\
\hline Valve surgery, $^{\zeta}$ & $15(50)$ & $16(50)$ \\
\hline Duration of procedure, $(\mathrm{SD})$, min & $194(18)$ & $170(10)$ \\
\hline Duration of $\mathrm{CPB},(\mathrm{SD})$, min & $116(12)$ & $92(7)$ \\
\hline Duration of cross clamp time, $(\mathrm{SD})$, min & $85(8)$ & $70(5)$ \\
\hline
\end{tabular}

function, as a possible determinant of the risk for atrial fibrillation after cardiac surgery. No effect of dexamethasone on LA ejection fraction or dimensions was found when compared with placebo. Also, dexamethasone did not significantly reduce the risk of PNAF.

Atrial fibrillation is a very common cardiovascular disease with a lifetime risk in the general Dutch population of 25$30 \%$ [21]. In the cardiac surgical population, its incidence is very high, being up to a third of all patients developing PNAF, and older age is still the only preoperative risk factor that has been consistently shown to predict the occurrence of PNAF $[2,3]$. Nonetheless, LA dimensions and function have been implicated to be predictors of PNAF in the postoperative setting in several studies [9, 10]. Preoperative LA-TEF was shown by Haffajee et al. to predict PNAF even better than LA volume or diameter [10]. Osranek et al. found that preoperative maximum LA volume $\left(32 \mathrm{ml} / \mathrm{m}^{2}\right)$ was an important predictor of PNAF [9]. However, Gibson et al. did not confirm this [6].

The mechanisms that contribute to the development of LA dysfunction after cardiac surgery are still not entirely clear. In patients with a positive fluid balance, the left atrium is often exposed to a higher preload postoperatively when compared with the preoperative period. Additionally, due to surgical
Table 2 Comparison of preoperative echocardiographic parameters in the dexamethasone and placebo groups $(N=44)$. Data shown in means $\pm \mathrm{SD}$

Abbreviations: $C I$ : confidence interval; $L A$ : left atrial; $N$ : number; $S D$ : standard deviation; $T E F$ : total ejection fraction

\begin{tabular}{llllll}
\hline $\begin{array}{l}\text { Ultrasound } \\
\text { parameter }\end{array}$ & $\begin{array}{l}\text { Dexamethasone } \\
(N=22)\end{array}$ & $\begin{array}{l}\text { Placebo } \\
(N=22)\end{array}$ & $\begin{array}{l}\text { Mean } \\
\text { difference }\end{array}$ & $\begin{array}{l}95 \% \text { CI of } \\
\text { difference }\end{array}$ & P Value \\
\hline LA-TEF, \% & $41.1(12.4)$ & $42.2(14.0)$ & -1.1 & -9.2 to 6.9 & 0.77 \\
LA diameter, cm & $4.2(0.7)$ & $4.3(0.8)$ & -0.1 & -0.6 to 0.4 & 0.11 \\
LA area, cm ${ }^{2}$ & $15.3(5.0)$ & $14.9(4.6)$ & 0.4 & -2.4 to 3.4 & 0.75 \\
LA max, ml & $43.0(19.6)$ & $42.8(18.6)$ & 0.2 & -11.4 to 11.9 & 0.97 \\
LA min, ml & $26.9(16.2)$ & $26.0(15.2)$ & 0.9 & -8.6 to 10.5 & 0.85 \\
\hline
\end{tabular}


Table 3 Comparison of postoperative echocardiographic parameters in the dexamethasone and placebo groups $(N=62)$. Data shown in means $\pm \mathrm{SD}$

\begin{tabular}{|c|c|c|c|c|c|}
\hline Ultrasound parameter & Dexamethasone $(N=30)$ & Placebo $(N=32)$ & Mean difference & $95 \%$ CI of Difference & $\mathrm{P}$ Value \\
\hline LA-TEF, \% & $36.4(11.9)$ & $40.2(7.9)$ & -3.8 & -9.0 to 1.4 & 0.15 \\
\hline LA diameter, $\mathrm{cm}$ & $4.6(0.8)$ & $4.3(0.9)$ & 0.3 & -0.2 to 0.7 & 0.19 \\
\hline LA area, $\mathrm{cm}^{2}$ & $16.0(5.0)$ & $16.4(5.9)$ & -0.4 & -3.1 to 2.4 & 0.81 \\
\hline LA max, ml & $31.0(12.3)$ & $31.3(13.9)$ & -0.3 & -7.0 to 6.3 & 0.91 \\
\hline LA min, ml & $19.7(10.7)$ & $18.8(9.8)$ & 0.9 & -4.3 to 6.2 & 0.73 \\
\hline LAA area, $\mathrm{cm}^{2}$ & $2.9(0.9)$ & $2.7(0.8)$ & 0.2 & -0.3 to 0.6 & 0.47 \\
\hline LAA velocity, $\mathrm{cm} / \mathrm{s}$ & $57.3(19.6)$ & $58.9(16.3)$ & -1.6 & -11.6 to 8.4 & 0.75 \\
\hline Left pulmonary vein $\mathrm{S} / \mathrm{D}$ ratio & $1.3(0.2)$ & $1.5(0.4)$ & -0.2 & -0.4 to 0.1 & 0.17 \\
\hline
\end{tabular}

Abbreviations: $C I$ : confidence interval; $L A$ : left atrial; $L A A$ : left atrial appendage; $N$ : number; $S / D$ ratio: systolic/diastolic ratio; $S D$ : standard deviation; $T E F$ : total ejection fraction

trauma, the left atrium is exposed to a systematic inflammatory response. The higher preload and the systemic inflammation may result in atrial dilatation and fibrotic infiltrates. These changes lead to temporary electromechanical and morphological changes in the atrial myocardium and can subsequently cause the formation of micro re-entrant pathways [14, 22]. Several anti-inflammatory drugs have been studied to reduce PNAF, e.g. statins and corticosteroids. Large observational studies and trials with statins for this indication have shown conflicting results, but in a recent systematic review it was concluded that statins have positive effects on protecting against PNAF [23-25]. In the current study the authors focused on investigating corticosteroids and their role in reducing PNAF. Corticosteroids are known to inhibit both inflammation and tissue changes in the heart, by suppressing inflammation-induced proliferation of myocardial fibroblasts as well as by preventing the activation of ERK1/2 and NF-кB [26, 27]. Several clinical studies have shown that intraoperative corticosteroids reduce the risk of PNAF and the consistency of these findings has recently been confirmed by several reviews and meta-analyses [17, 25, 28].

In this study, dexamethasone did not have any demonstrable effects on TEF, LA and LAA dimensions. These findings could be due to the fact that we have missed an actual effect of dexamethasone because the number of patients included in this study was relatively small. On the other hand, other factors could have contributed to missing an effect. The relatively high age of the study population (mean age of 70) could have contributed, since ageing often leads to a permanent loss of myocardial muscle fibres and an increase in fibrosis, resulting in irreversible atrial remodelling which may not be influenced by dexamethasone [29]. Also, LA dimensions and function strongly depend on loading conditions. Most patients are intravascularly well loaded after the operation and most are on inotropic support, which might have confounded the effect of dexamethasone on the left atrium. However, it seems very reasonable to assume that dexamethasone does not have an effect on LA-TEF at all. Moreover, no association between LA-TEF and PNAF could be demonstrated, and the incidence of PNAF was not reduced by dexamethasone. The latter is contradictory to several previous studies looking at the effects of steroids on PNAF, although it is in line with the findings of the DECS trial, where no effect of treatment on PNAF could be demonstrated in 4482 patients.

This study demonstrates that PNAF is a complex arrhythmia with multiple mechanisms underlying its pathology, whereby atrial remodelling through an inflammatory pathway may only play a minor role. Future studies should investigate atrial biopsies to attempt to elucidate subtle and delicate pathophysiological inflammatory and microfibrotic changes in the atrial myocardium, as well as the effects on this of antiinflammatory agents.

\section{Strengths and limitations}

The strengths of this study include the randomised doubleblinded placebo-controlled design, which allowed for strict distribution of the patient's clinical and demographic characteristics. Continuous Holter monitoring for 5 days postoperatively ensured that all PNAF episodes could be detected for accurate quantification.

A limitation of this study is that the sample size was small. Another limitation is that with transoesophageal echocardiography there might be an underestimation of LA dimension. As this holds for both study groups, and for the measurements obtained before and after surgery, we do not expect an effect on the study results obtained. Another limitation is the fact that LA-TEF was measured and calculated based on transoesophageal echocardiography images, using the same methods and mathematical formula that have been used in previous studies using transthoracic echocardiography. Although these methods have not been well validated in transoesophageal echocardiography, the potential error is probably the same for both groups. 


\section{Conclusion}

Intraoperative high-dose dexamethasone did not have any protective effect on postoperative LA-TEF or dimension and did not reduce the risk of PNAF in cardiac surgical patients.

Acknowledgments The authors would like to thank the Physiological ECG Services for providing the Holter devices for the study, and for analysing the Holter data. This work was supported by the Netherlands Organisation for Health Research and Development (ZonMw) [grant number 80-82310-98-08607), the Dutch Heart Foundation [grant number 2007B125] and the European Association of Cardiothoracic Anaesthesiologists [2010-Research Grants]. None were involved in data collection, analysis and interpretation.

Trial registration This trial is registered with ClinicalTrials.gov, number NCT00293592.

Conflict of interest none declared.

Open Access This article is distributed under the terms of the Creative Commons Attribution License which permits any use, distribution, and reproduction in any medium, provided the original author(s) and the source are credited.

\section{References}

1. Attaran S, Shaw M, Bond L, et al. Atrial fibrillation postcardiac surgery: a common but a morbid complication. Interact Cardiovasc Thorac Surg. 2011;12:772-7.

2. Auer J, Weber T, Berent R, et al. Postoperative atrial fibrillation independently predicts prolongation of hospital stay after cardiac surgery. J Cardiovasc Surg (Torino). 2005;46:583-8.

3. Mathew JP, Fontes ML, Tudor IC, et al. A multicenter risk index for atrial fibrillation after cardiac surgery. JAMA. 2004;291:1720-9.

4. Acil T, Colkesen Y, Turkoz R, et al. Value of preoperative echocardiography in the prediction of postoperative atrial fibrillation following isolated coronary artery bypass grafting. Am J Cardiol. 2007;100: 1383-6.

5. Benedetto U, Melina G, Roscitano A, et al. Clinical utility of tissue Doppler imaging in prediction of atrial fibrillation after coronary artery bypass grafting. Ann Thorac Surg. 2007;83:83-8.

6. Gibson PH, Croal BL, Cuthbertson BH, et al. Use of preoperative natriuretic peptides and echocardiographic parameters in predicting new-onset atrial fibrillation after coronary artery bypass grafting: a prospective comparative study. Am Heart J. 2009;158:244-51.

7. Leung JM, Bellows WH, Schiller NB. Impairment of left atrial function predicts post-operative atrial fibrillation after coronary artery bypass graft surgery. Eur Heart J. 2004;25:1836-44.

8. Nakai T, Lee RJ, Schiller NB, et al. The relative importance of left atrial function versus dimension in predicting atrial fibrillation after coronary artery bypass graft surgery. Am Heart J. 2002;143:181-6.

9. Osranek M, Fatema K, Qaddoura F, et al. Left atrial volume predicts the risk of atrial fibrillation after cardiac surgery: a prospective study. J Am Coll Cardiol. 2006;48:779-86.

10. Haffajee JA, Lee Y, Alsheikh-Ali AA, et al. Pre-operative left atrial mechanical function predicts risk of atrial fibrillation following cardiac surgery. JACC Cardiovasc Imaging. 2011;4:833-40.
11. Antoniades C, Demosthenous M, Reilly S, et al. Myocardial redox state predicts in-hospital clinical outcome after cardiac surgery effects of short-term pre-operative statin treatment. J Am Coll Cardiol. 2012;59:60-70.

12. Kim YM, Kattach H, Ratnatunga C, et al. Association of atrial nicotinamide adenine dinucleotide phosphate oxidase activity with the development of atrial fibrillation after cardiac surgery. J Am Coll Cardiol. 2008;51:68-74.

13. Ramlawi B, Otu H, Mieno S, et al. Oxidative stress and atrial fibrillation after cardiac surgery: a case-control study. Ann Thorac Surg. 2007;84:1166-72. discussion 72-3.

14. Frustaci A, Chimenti C, Bellocci F, et al. Histological substrate of atrial biopsies in patients with lone atrial fibrillation. Circulation. 1997;96:1180-4.

15. Chaney MA. Corticosteroids and cardiopulmonary bypass : a review of clinical investigations. Chest. 2002;121:921-31.

16. Dieleman JM, Nierich AP, Rosseel PM, et al. Intraoperative highdose dexamethasone for cardiac surgery: a randomized controlled trial. JAMA. 2012;308:1761-7.

17. Whitlock RP, Chan S, Devereaux PJ, et al. Clinical benefit of steroid use in patients undergoing cardiopulmonary bypass: a meta-analysis of randomized trials. Eur Heart J. 2008;29:2592-600.

18. Halonen J, Halonen P, Jarvinen O, et al. Corticosteroids for the prevention of atrial fibrillation after cardiac surgery: a randomized controlled trial. JAMA. 2007;297:1562-7.

19. Eshoo S, Ross DL, Thomas L. Evaluation of left atrial size on transoesophageal echocardiography: what is the best measure? Heart Lung Circ. 2008;17:100-6.

20. Singh H, Jain AC, Bhumbla DK, et al. Comparison of left atrial dimensions by transesophageal and transthoracic echocardiography. Echocardiog. 2005;22:789-96.

21. Leening MJ, Siregar S, Vaartjes I, et al. Heart disease in the Netherlands: a quantitative update. Neth Heart J. 2014;22:310.

22. Chung MK, Martin DO, Sprecher D, et al. C-reactive protein elevation in patients with atrial arrhythmias: inflammatory mechanisms and persistence of atrial fibrillation. Circulation. 2001;104:2886-91.

23. Folkeringa RJ, Tieleman RG, Maessen JG, et al. Statins do not reduce atrial fibrillation after cardiac valvular surgery: a single centre observational study. Neth Heart J. 2011;19:17-23.

24. Hartholt NL, Rettig TC, Schijffelen M, et al. Preoperative statin therapy and infectious complications in cardiac surgery. Neth Heart J. 2014. doi:10.1007/s12471-014-0581-5.

25. Jacob KA, Nathoe HM, Dieleman JM, et al. Inflammation in newonset atrial fibrillation after cardiac surgery: a systematic review. Eur J Clin Invest. 2014;44(4):402-28.

26. He YH, Zhang HN, Zhang GP, et al. A physiological concentration of glucocorticoid inhibits the pro-inflammatory cytokine-induced proliferation of adult rat cardiac fibroblasts: roles of extracellular signalregulated kinase 1/2 and nuclear factor-kappaB. Clin Exp Pharmacol Physiol. 2011;38:739-46.

27. Nebelsiek T, Beiras-Fernandez A, Kilger E, et al. Routine use of corticosteroids to prevent inflammation response in cardiac surgery. Recent Pat Cardiovasc Drug Discov. 2012;7:170-4.

28. Cappabianca G, Rotunno C, de Luca Tupputi Schinosa L, et al. Protective effects of steroids in cardiac surgery: a meta-analysis of randomized double-blind trials. J Cardiothorac Vasc Anesth. 2011;25:156-65.

29. Goette A, Juenemann G, Peters B, et al. Determinants and consequences of atrial fibrosis in patients undergoing open heart surgery. Cardiovasc Res. 2002;54:390-6. 